COEFFIGIENTE DI TEMPERAT. DELLE SOLUZIONI DI SALI ECC. 359

Oltre che al cloruro ferrico, si potrebbe ricorrere al cloruro di oro, al cloruro di platino, al cloruro di mercurio ; è presumibile peró che il punto neutro debba variare anche al variare del sale, e specialmente al variare del suo stato per quanto riguarda la maggiore o minor secchezza del sale stesso.

I. Bosi.

\title{
SULLA DOPPIA RIFRAZIONE ELETTRICA DEL LEGNO,
}

del Prof. D. MAZZO'TTO.

(Largo sunto dejla Nota pubblicuta nei Renđiconti della R. Accademia dei lincei. Vol. $6^{0}, 2^{0}$ sem., serie 5 , fasc. $3^{0}$ ).

L'A. si prefgrge di determinare, per rarie qualitá di legno, $i$ due indici principali di rifrazione elettrica corrispondenti a vibrazioni perpendicolari o parallele alle fibre.

L' apparecchio msato per le esperienze era quello stesso di Lecher con alcune modificazioni, fra cui due appendici aggiunte ai fili secondari. Nelle esperienze si fece sempre uso della vibrazione secondarià.

I blocchi di legno da studiare aveano la forma di parallelepipedo cogli spigoli più lungi disposti parallelamente e simmetricamente l'ispetto ai fili secondari, ed erano costituiti da tre parallelepipedi di egual lunghezza ed altezza, posti l'uno accanto all' altro e tenuti serrati da morse di legno. Il parallelepipedo centrale avea la larghozza di $6 \mathrm{~cm}$, uguale cioè alla distanza dei fili secondari, o questi erano incastrati in una piccola scanalatura praticata lungo le facce laterali di esso. Due ponti rettilinei $p_{1}$ e $p_{2}$ metterano in comunicazione i fili secondari all' entratat ed all'uscita del bloceo di legno.

Col mezzo d'una funicella si lacea scorrele un $3^{\prime \prime}$ ponte $p_{3}$ lungo il tratto di fili secondari uscenti dal legno, e fra esso ed il ponte $p_{2}$ era collocato l'asploratore, costituito da due corti filj metallici flssati sopra uni taroletta i quali, con una estremitit, rivolta ad uncinetto, si aggrappano ai fili secondari e ripiegandosi poi opizzontamente al angolo retto terminano in due punte distanif circa un centimetro l' una dall' altra. Se, 
nell' oscurita, si appressano a queste punte le estremiti delle dita, si scorgono, se il sistema vibrante é prossimo alla risonanza, delle scintilline che presentano un massimo quando la risonanza è perfetta.

Una determinazione consistera nel misurare la lunghezza, $\lambda \mid 2$, della semionda nell' aria che corrisponde alla semionda $\lambda_{1} \mid 2$ nel legno, limitata fra i ponti $p_{1}$ e $p_{2}$. Per far cio, lasciati fissi i ponti $p_{1} p_{2}$, si faceva scorrere il ponte $p_{3}$ finchè com parivano delle scintille all esploratore, e si cercava la lunghezza da dare alle appendici, perchè queste scintille raggiunsero il massimo di intensita; si notarano allora le posizioni dei tre ponti e si allontanava ancora il ponte $p_{3}$ da $\mu_{2}$, finchè in una nuova posizione $p_{4}$ si areva di nuoro un massimo scintillamento all esploratore. Si aveva allora la risonanza fra le quattro semionde comprese fira l'eccitatore e le posizioni dei ponti. Dalle posizioni dei ponti, si determinano, fatte le debite correzioni, i ralori $\lambda \mid 2$ e $\lambda_{1} \mid 2$ e da questi il ralore dell' indice di rifrazione $n=\lambda \mid \lambda_{1}$.

Le correzioni consistono nel determinare la quantità costante $\delta$, dovuta alla presenzal dei ponti e dell' esploratore, da aggiungersi alla lunghezza $p_{2} \nu_{3} \mathrm{pel}^{2}$ avere la lunghezza $\lambda \mid 2$ nell' aria, e la quantita pure costante $\delta_{1}$, dovuta alla presenza dei ponti terminali, da aggiungersi alla lungheza $p_{1} p_{2}$ per avere la lunghezza $\lambda_{1} \mid 2$ nel legno.

Per meglio eliminare l' influenza degli errori di osservazione, le determinazioni di $n$ si fecero in massima tre volte in condizioni differenti, cioè cambiando di volta in volta la posizione del blocco di legno lungo i fili secontari, cosi che fosse differente in ciascun caso la lunghezza delle appendici occorrenti per la risonanza.

Prima di applicare il metodo alle misure definitive, l' A. volle studiare esperimentalmente quale fosse l'estensione da darsi alla massa del legrno attorno ai fili secondarj, perchè i risultati fossero praticamente equiralenti a quelli che si arrebbero con una massa di leuno estesa all' infinito.

Determinó perció il ralore $n$ usando lamine di abete di spessore (nel senso verticale) man mano crescente. Dai risultati ottenuti si ricavo che l'indice di rifrazione riesce piu basso del 
vero usando lamine dello spessore di soli due centimetri, ma che gia collo spessore di $4 \mathrm{~cm}$. si ottengono valori poco differenti a quelli che si ottengono con lamine di 18 e 27 centimetri di spessore. Quindi ho spossore di circa l: cm. che l'A. ha usato ordinarianente pui ritenersi sulficiente.

Le esperienze furono fatte su parecchie qualita di legno. Le tre posizioni in cui erano disposte le fibre dei legni rispetto ai lili secondari sono:

$1^{2}$ Longitudinale: quanto le fore ozano parallele ai fili e quindi la propagazione seguira parallelamente alle fibre e la vibrazione perpendicolarmente ad esse.

2" Trasversale a fitre oriszontali: quando le tibre emo perpendicolari ai fili ma parallele al loro piano, e quindi la propagazione perpendicolare alle fibre e la vibrazione par. rallela ad esse.

$3^{2}$ Trasuersale a fibre verticali: quando le fibre erano perpendicolari al piano lei flli, e guindi tanto la propagazione che la ribrazione orano perpendicolari alle fibre.

I parallelepipedi di ciascuna qualita di lesno erano tratti da uno stesso tarolone o ravicello, el erano di un sol pezzo quelli"longitudinali e di pi perri (da :3 a 5) insieme incollati, quelli trasrersali. In molti casi (abete II, pero, pitch-pine, elce), i parallelepipedi erano stati composti colla stessa masia di legno, segata ed incollata in modi differenti in modo cla dare alle fibre la direzione roluta, e ció jer togliere il dubbio che le differenze fossero dorute alle diversitit che pur offrire il legno in parti differenti di uno stesso ceppo.

Nella tabella seguente sono raccolte, caso per caso, le medie degli indici di rifrazione trovati, insieme ai ralori dei pesi specifici dei rispetivi logni, determinati facendo il rapporto lia il péso dei parallelepipedi di cjacum legno ed il loro rolume, calcolato geometricamente. 


\begin{tabular}{|c|c|c|c|c|}
\hline & \multicolumn{3}{|c|}{ INDICI III RIFRAZIONE } & \multirow{4}{*}{ 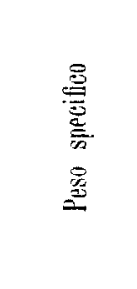 } \\
\hline & \multirow{3}{*}{$\begin{array}{c}\text { longitudi- } \\
\text { nale }\end{array}$} & \multicolumn{2}{|c|}{ ropagazione } & \\
\hline & & \multicolumn{2}{|c|}{ trasversale } & \\
\hline & & $\begin{array}{c}\text { fibre } \\
\text { verticali }\end{array}$ & $\begin{array}{c}\text { fibre } \\
\text { orizzontali }\end{array}$ & \\
\hline Abete $\left\{\begin{array}{l}\text { I } \\
\text { II } \\
\text { III } \\
\text { IV }\end{array}\right.$ & $\begin{array}{c}1,540 \\
1,596 \\
- \\
-\end{array}$ & $\begin{array}{l}1,646 \\
1,679 \\
1,604 \\
1,721\end{array}$ & $\begin{array}{l}\overline{1,730} \\
1,657 \\
1,832\end{array}$ & $\begin{array}{l}0,458 \\
0,463 \\
0,442 \\
0,471\end{array}$ \\
\hline Media & 1,568 & 1,663 & 1,739 & 0,458 \\
\hline $\begin{array}{l}\text { Pino } \\
\text { Pioppo } \\
\text { Pitch-Pine } \\
\text { Pero } \\
\text { Noce }\end{array}$ & $\begin{array}{l}\overline{1,585} \\
1,759 \\
1,781 \\
1,875\end{array}$ & $\begin{array}{l}1,590 \\
1,621 \\
1,890 \\
1,794 \\
-\end{array}$ & $\begin{array}{l}1,635 \\
\overline{1}, 869 \\
1,800 \\
1,938\end{array}$ & $\begin{array}{l}0,441 \\
0,567 \\
0,636 \\
0,659 \\
0,725\end{array}$ \\
\hline Olivo & $\begin{array}{c}2,051 \\
(a)\end{array}$ & $\begin{array}{c}1,903 \\
(b)\end{array}$ & - & $\begin{array}{l}\text { a) } 1,004 \\
\text { b) } 0,958\end{array}$ \\
\hline Elce & 2,211 & 2,252 & $2,2,36$ & 1,238 \\
\hline
\end{tabular}

Dalle presenti esperienze si possono trarre le conclusioni seguenti :

1a $L$ 'indice di rifrazione elettrica varia notevolmente da una specie all' altra di legno e cresce colla densità entro i limiti 1, ó40 (abete: densità $=0,450$ ) e 2,244 (elce: densità $=1,238$ ).

2 a Nello stesso legno la vibrazione elettrica perpendicolare alle fibre si propaga pia velocemente della vibrazione parallela alle fibre, quindi nel primo caso l'indice di rifrazione è minore che nel secondo.

$3^{\text {a }}$ La differenza dei due indici è minore nei legni più densi e compatti che in quelli piu leggeri.

I. Bosi. 